\title{
Existence of multi-valued solutions with asymptotic behavior of parabolic Monge-Ampère equation
}

\section{Limei Dai*}

\section{"Correspondence:} Imdai@wfu.edu.cn School of Mathematics and Information Science, Weifang University, Weifang, Shandong 261061, China

\begin{abstract}
In this paper, we extend the results of multi-valued solutions of elliptic Monge-Ampère equation to parabolic Monge-Ampère equation. We use the Perron method to prove the existence of multi-valued solutions with asymptotic behavior at infinity of parabolic Monge-Ampère equation. Moreover, we prove that the multi-valued solution is continuous in the whole space.
\end{abstract}

MSC: $35 \mathrm{~K} 96 ; 35 \mathrm{~B} 40$

Keywords: parabolic Monge-Ampère equation; multi-valued solutions; asymptotic behavior; Perron method

\section{Introduction}

We consider the multi-valued solutions of the parabolic Monge-Ampère equation

$$
-u_{t} \operatorname{det}\left(D^{2} u\right)=1,
$$

where $u=u(x, t)$ is convex in $x$ and nonincreasing in $t, x \in \mathbb{R}^{n}, t \in \mathbb{R}$, and $D^{2} u=D_{x}^{2} u$ denotes the Hessian of $u$ with respect to the variable $x$. The parabolic equation (1.1) was first introduced by Krylov [1] together with the other parabolic versions of elliptic MongeAmpère equation; see [2] for a complete description and related results. Because of its importance in stochastic theory, Krylov further discussed (1.1) in [3]. It is also relevant in the study of deformation of surfaces by Gauss-Kronecker curvature [4] and in a maximum principle for parabolic equations [5]. Tso [5] pointed out that (1.1) is the most appropriate parabolic version of the elliptic Monge-Ampère equation $\operatorname{det}\left(D^{2} u\right)=f$ in the proof of the Aleksandrov-Bakelman maximum principle of second order parabolic equations.

There is a vast literature on the parabolic Monge-Ampère equation (1.1); see [2, 6-11] etc. In particular, Gutiérrez and Huang [7] obtained a generalization of a theorem by Calabi. Wang and Wang [9] proved the existence of viscosity solutions by the approximation procedure and the nonlinear perturbation method, and they also obtained the regularity of the viscosity solutions.

From the theory of analytic functions, we know that the typical two dimensional examples of multi-valued harmonic functions are

$$
\begin{aligned}
& u_{1}(z)=\operatorname{Re}\left(z^{\frac{1}{k}}\right), \quad z \in \mathbb{C} \backslash\{0\}, \\
& u_{2}(z)=\operatorname{Arg}(z), \quad z \in \mathbb{C} \backslash\{0\}
\end{aligned}
$$

(c) 2015 Dai; licensee Springer. This is an Open Access article distributed under the terms of the Creative Commons Attribution License (http://creativecommons.org/licenses/by/4.0), which permits unrestricted use, distribution, and reproduction in any medium, provided the original work is properly credited. 
and

$$
u_{3}(z)=\operatorname{Re}(\sqrt{(z-1)(z+1)}), \quad z \in \mathbb{C} \backslash\{ \pm 1\}
$$

where $\mathbb{C}$ is the plural set.

There are many results as regards the multi-valued solutions. Evans [12] and Caffarelli [13] studied the multi-valued harmonic functions. Evans [12] has proved that the conductor potential of a surface with minimal capacity is a double-valued harmonic function. In [13], Caffarelli proved the Hölder continuity of the multi-valued harmonic functions. Jin et al. [14-16] employed a level set method for the computation of multi-valued geometric solutions to general quasilinear PDEs and multi-valued physical observables to the semiclassical limit of the Schrödinger equations. In 2006, Caffarelli and Li [17] investigated the multi-valued solutions of the Monge-Ampère equations. They first introduced the geometric situation of the multi-valued solutions and then obtained the existence, boundedness, regularity and the asymptotic behavior at infinity of the multi-valued viscosity solutions. Recently, the author and Bao [18] investigated the multi-valued solutions of the Hessian equations. The author [19] also studied the finitely valued and infinitely valued solutions to the parabolic Monge-Ampère equation $-u_{t} \operatorname{det}\left(D^{2} u\right)=f$. For more detailed introduction of the multi-valued solutions and other models in nonlinear PDEs, see $[17,18]$ and [20]. In this paper, we extend some results of the multi-valued solutions in [17] to the parabolic Monge-Ampère equation $-u_{t} \operatorname{det}\left(D^{2} u\right)=f$.

The geometric situation of the multi-valued solutions to the elliptic equations can be found in [17]. The geometric situation of the multi-valued solutions to the parabolic equations is the following. Let $n \geq 2, \mathbb{R}_{T}^{n+1}=\mathbb{R}^{n} \times(0, T], T>0, \Omega \subset \mathbb{R}^{n}$ be a bounded strictly convex domain with smooth boundary $\partial \Omega$. Suppose $\Omega$ is homeomorphic in $\mathbb{R}^{n}$ to an $n$ dimensional closed disc, i.e., there exists a homeomorphism $\psi: \mathbb{R}^{n} \rightarrow \mathbb{R}^{n}$ such that $\psi(\Omega)$ is an $n$ dimensional closed disc. Let $\Omega_{t_{0}}=\Omega \times\left\{t_{0}\right\}, 0<t_{0}<T$. Then $\Omega_{t_{0}}$ divides $Q=\Omega \times(0, T]$ into two parts, denoted as $Q^{+}=\Omega \times\left(t_{0}, T\right]$ and $Q^{-}=\Omega \times\left(0, t_{0}\right]$. We use the convention that going through $\Omega_{t_{0}}$ from $Q^{-}$to $Q^{+}$denote the positive direction. Let $\Gamma=\partial \Omega \times\left\{t_{0}\right\}, \mathbb{Z}$ be the set of integers, and

$$
G=\left(\mathbb{R}_{T}^{n+1} \backslash \Gamma\right) \times \mathbb{Z}
$$

denote a covering of $\mathbb{R}_{T}^{n+1} \backslash \Gamma$ with the following standard parameterization: fixing an $\left(x^{*}, t^{*}\right) \in \mathbb{R}_{T}^{n+1} \backslash \Gamma$ and connecting $\left(x^{*}, t^{*}\right)$ by a smooth curve in $\mathbb{R}_{T}^{n+1} \backslash \Gamma$ to a point $(x, t) \in$ $\mathbb{R}_{T}^{n+1} \backslash \Gamma$. If the curve goes through $\Omega_{t_{0}}, m \geq 0$ times in the positive direction, then we arrive at $(x, t, m)$ in $G$. If the curve goes through $\Omega_{t_{0}}, m \geq 0$ times in the negative direction, then we arrive at $(x, t,-m)$ in $G$.

For $k \in \mathbb{Z}, k \geq 2$, we introduce an equivalence relation ' $\sim_{k}$ ' in $G$ as follows: $(x, t, m)$ and $(\tilde{x}, \tilde{t}, j)$ in $G$ are ' $\sim$ ' equivalent if $x=\tilde{x}, t=\tilde{t}$ and $m-j$ is an integer multiple of $k$. Let

$$
G_{k}=G / \sim_{k}
$$

denote the $k$-sheet cover of $\mathbb{R}_{T}^{n+1} \backslash \Gamma$.

The distance in $G_{k}$ is defined as follows: For any $(x, t, m),(\tilde{x}, \tilde{t}, j) \in G_{k}$, suppose $l((x, t, m)$, $(\tilde{x}, \tilde{t}, j))$ is a smooth curve in $G_{k}$ that connects the points $(x, t, m)$ and $(\tilde{x}, \tilde{t}, j)$, and let 
$|l((x, t, m),(\tilde{x}, \tilde{t}, j))|$ denote the length of the curve. Define

$$
d((x, t, m),(\tilde{x}, \tilde{t}, j))=\inf _{l}|l((x, t, m),(\tilde{x}, \tilde{t}, j))|
$$

where the inf is taken over all smooth curves connecting $(x, t, m)$ and $(\tilde{x}, \tilde{t}, j)$. Then $d((x, t, m),(\tilde{x}, \tilde{t}, j))$ is a distance.

Definition 1.1 We say a function $u$ is continuous at $(x, t, m)$ in $G_{k}$ if

$$
\lim _{d((x, t, m),(\tilde{x}, \tilde{t}, j)) \rightarrow 0} u(\tilde{x}, \tilde{t}, j)=u(x, t, m)
$$

and $u \in C^{0}\left(G_{k}\right)$ if for any $(x, t, m) \in G_{k}, u$ is continuous at $(x, t, m)$.

Similarly we say a function $u \in C^{2,1}\left(G_{k}\right)$ if $D_{x}^{i} D_{t}^{j} u(i+2 j \leq 2)$ is continuous at $(x, t, m)$ for any $(x, t, m) \in G_{k}$.

Definition 1.2 A function $u \in C^{0}(Q)$ is called parabolically convex in $Q$, if $u$ is convex in $x$ and nonincreasing in $t$.

Our purpose of this paper is to study the existence of multi-valued viscosity solutions with asymptotic behavior of the parabolic Monge-Ampère equation (1.1). We shall extend the results of the elliptic equations to the parabolic equation (1.1). The main result of this paper is the existence theorem of multi-valued solutions with prescribed asymptotic behavior at infinity.

Theorem 1.1 Let $n \geq 3, k \geq 2$ and $\Omega=B_{1}(0)=\{x:|x| \leq 1\}$. Then for any $c_{m} \in \mathbb{R}, 1 \leq m \leq k$, there exist some constant $\beta$ and a locally parabolically convex viscosity solution $u \in C^{0}\left(G_{k}\right)$ of

$$
-u_{t} \operatorname{det}\left(D^{2} u\right)=1, \quad(x, t, m) \in G_{k}
$$

satisfying

$$
\begin{aligned}
& \limsup _{|x| \rightarrow \infty}\left[|x|^{n-2}\left|u(x, t, m)-\left(-t+\frac{1}{2}|x|^{2}+c_{m}\right)\right|\right]<\infty, \\
& \lim _{(x, t) \rightarrow\left(\bar{x}, t_{0}\right)} u(x, t, m)=\beta, \quad\left(\bar{x}, t_{0}\right) \in \Gamma .
\end{aligned}
$$

This paper is arranged as follows. In Section 2, we give some lemmas and in Section 3, we shall prove Theorem 1.1.

\section{Preliminaries}

For the reader's convenience, we first give the definition of the viscosity solutions to the parabolic Monge-Ampère equation

$$
-u_{t} \operatorname{det}\left(D^{2} u\right)=1 \quad \text { in } G_{k} \text {. }
$$

See also [19]. 
Definition 2.1 A function $u(x, t, m) \in C^{0}\left(G_{k}\right)$ is called a viscosity subsolution of (2.1), if $u(x, t, m)$ is locally parabolically convex, and for any $\sigma \in C^{2,1}\left(G_{k}\right)$, any $(\tilde{x}, \tilde{t}) \in \mathbb{R}_{T}^{n+1} \backslash \Gamma$, satisfying

$$
u(\tilde{x}, \tilde{t}, m)=\sigma(\tilde{x}, \tilde{t}, m), \quad u(x, t, m) \leq \sigma(x, t, m), \quad(x, t, m) \in G_{k}, t \leq \tilde{t}
$$

we have

$$
-\sigma_{t}(\tilde{x}, \tilde{t}, m) \operatorname{det}\left(D^{2} \sigma(\tilde{x}, \tilde{t}, m)\right) \geq 1
$$

A function $u(x, t, m) \in C^{0}\left(G_{k}\right)$ is called a viscosity supersolution of $(2.1)$, if $u(x, t, m)$ is locally parabolically convex, and for any $\sigma \in C^{2,1}\left(G_{k}\right)\left(D^{2} \sigma(\tilde{x}, \tilde{t}, m)>0\right)$, any $(\tilde{x}, \tilde{t}) \in \mathbb{R}_{T}^{n+1} \backslash \Gamma$, satisfying

$$
u(\tilde{x}, \tilde{t}, m)=\sigma(\tilde{x}, \tilde{t}, m), \quad u(x, t, m) \geq \sigma(x, t, m), \quad(x, t, m) \in G_{k}, t \leq \tilde{t},
$$

we have

$$
-\sigma_{t}(\tilde{x}, \tilde{t}, m) \operatorname{det}\left(D^{2} \sigma(\tilde{x}, \tilde{t}, m)\right) \leq 1 .
$$

A function $u(x, t, m) \in C^{0}\left(G_{k}\right)$ is called a viscosity solution of $(2.1)$, if $u(x, t, m) \in C^{0}\left(G_{k}\right)$ is both a viscosity subsolution and a viscosity supersolution of (2.1).

Let $Q=\Omega \times(0, T], \partial_{p} Q=\partial \Omega \times(0, T) \cup \bar{\Omega} \times\{0\}$ be the parabolic boundary of $Q$, and $S Q=\partial \Omega \times(0, T)$ be the side boundary of $Q, \bar{S} Q=\partial \Omega \times[0, T]$. The following lemmas and remark can be found in [19].

Lemma 2.1 Let $\Omega^{\prime} \subset \subset \mathbb{R}^{n}$ be a bounded strictly convex domain, $0 \leq \bar{t}<T, D^{\prime}=\Omega^{\prime} \times(\bar{t}, T]$, $f \in C^{0}\left(\mathbb{R}_{T}^{n+1}\right)$. Assume that $u \in C^{0}\left(\mathbb{R}_{T}^{n+1}\right), v \in C^{0}\left(\bar{D}^{\prime}\right)$ satisfy respectively

$$
\begin{aligned}
& -u_{t} \operatorname{det}\left(D^{2} u\right) \geq f(x, t) \quad \text { in } \mathbb{R}_{T}^{n+1}, \\
& -v_{t} \operatorname{det}\left(D^{2} v\right) \geq f(x, t) \quad \text { in } D^{\prime}
\end{aligned}
$$

and

$$
\begin{array}{ll}
u=v & \text { on } \partial_{p} D^{\prime}, \\
u \leq v & \text { on } \bar{D}^{\prime} .
\end{array}
$$

Set

$$
w(x, t)= \begin{cases}u(x, t) & \text { in } \mathbb{R}_{T}^{n+1} \backslash D^{\prime} \\ v(x, t) & \text { in } D^{\prime}\end{cases}
$$

Then $w \in C^{0}\left(\mathbb{R}_{T}^{n+1}\right)$ and it satisfies in the viscosity sense

$$
-w_{t} \operatorname{det}\left(D^{2} w\right) \geq f(x, t) \quad \text { in } \mathbb{R}_{T}^{n+1}
$$


Remark 2.1 If $\bar{t}=0$, then we only need $u=v$ on $\bar{S} D^{\prime}$ in condition (2.2).

Lemma 2.2 Let $\Omega^{\prime} \subset \mathbb{R}^{n}$ be a bounded, strictly convex domain, $\partial \Omega^{\prime} \in C^{2}, Q^{\prime}=\Omega^{\prime} \times(0, T]$, $\bar{S} Q^{\prime}=\partial \Omega^{\prime} \times[0, T], w(x, t) \in C^{2,1}\left(\bar{Q}^{\prime}\right)$ and for $x \in \bar{\Omega}^{\prime}, w_{t}(x, t)=-1$. Then there exists some constant $C$, depending only on $n, w, Q^{\prime}$, such that, for any $\eta \in \partial \Omega^{\prime}, 0 \leq \lambda \leq T$, there exists $\bar{x}(\eta, \lambda) \in \mathbb{R}^{n}$ satisfying

$$
|\bar{x}(\eta, \lambda)| \leq C
$$

and

$$
p_{\eta, \lambda}(x, t)<w(x, t) \quad \text { on } \bar{Q}^{\prime} \backslash\{\eta, \lambda\},
$$

where

$$
p_{\eta, \lambda}(x, t)=w(\eta, \lambda)+\frac{1}{2}\left[|x-\bar{x}(\eta, \lambda)|^{2}-|\eta-\bar{x}(\eta, \lambda)|^{2}\right]-(t-\lambda), \quad(x, t) \in \mathbb{R}_{T}^{n+1} .
$$

Lemma 2.3 Suppose that $f \in C^{0}(Q)$ is nonnegative. Let $\mathbb{S}$ be a nonempty family of a subsolution of

$$
-u_{t} \operatorname{det}\left(D^{2} u\right)=f \quad \text { in } Q
$$

and

$$
u(x, t)=\sup \{v(x, t) \mid v \in \mathbb{S}\} \quad \text { for }(x, t) \in Q
$$

then $u$ is a viscosity subsolution of (2.4).

\section{Existence of multi-valued solutions with asymptotic behavior}

In this section, we will prove Theorem 1.1.

Proof We divide the proof into five steps.

Step 1 . We construct a viscosity subsolution of (1.2).

Let

$$
\phi(x, t)=-t+\frac{1}{2}|x|^{2}, \quad(x, t) \in \mathbb{R}^{n+1} .
$$

By Lemma 2.2, for any $\eta \in \partial \Omega, 0 \leq \lambda \leq T$, there exists $\bar{x}(\eta, \lambda) \in \mathbb{R}^{n},|\bar{x}(\eta, \lambda)|<\infty$ such that

$$
p_{\eta, \lambda}(x, t)<\phi(x, t) \quad \text { on } \bar{Q} \backslash\{\eta, \lambda\},
$$

where

$$
p_{\eta, \lambda}(x, t)=\phi(\eta, \lambda)+\frac{1}{2}\left(|x-\bar{x}(\eta, \lambda)|^{2}-|\eta-\bar{x}(\eta, \lambda)|^{2}\right)-(t-\lambda), \quad(x, t) \in \mathbb{R}_{T}^{n+1} .
$$

Then for any $\eta \in \partial \Omega, 0 \leq \lambda \leq T$,

$$
\begin{aligned}
& p_{\eta, \lambda}(\eta, \lambda)=\phi(\eta, \lambda), \\
& p_{\eta, \lambda}(x, t) \leq \phi(x, t) \quad \text { on } \bar{Q}
\end{aligned}
$$


and

$$
-\left(p_{\eta, \lambda}\right)_{t} \operatorname{det}\left(D^{2}\left(p_{\eta, \lambda}\right)\right)=1 \quad \text { in } \mathbb{R}_{T}^{n+1}
$$

Define

$$
p(x, t):=\sup _{\substack{\eta \in \partial \Omega \\ 0 \leq \lambda \leq T}} p_{\eta, \lambda}(x, t) \quad \text { in } \mathbb{R}_{T}^{n+1}
$$

Then $p$ satisfies

$$
\begin{aligned}
& p(x, t) \leq \phi(x, t) \quad \text { on } \bar{Q} \\
& p(x, t)=\phi(x, t) \quad \text { on } S Q
\end{aligned}
$$

and in the viscosity sense,

$$
-p_{t} \operatorname{det}\left(D^{2} p\right) \geq 1 \quad \text { in } \mathbb{R}_{T}^{n+1} \text {. }
$$

Set

$$
W(x, t)= \begin{cases}\phi(x, t) & \text { in } Q \\ p(x, t) & \text { in } \mathbb{R}_{T}^{n+1} \backslash Q\end{cases}
$$

By (3.2), $W \in C^{0}\left(\mathbb{R}_{T}^{n+1}\right)$. According to (3.1) and Remark 2.1, $W$ is parabolically convex and satisfies in the viscosity sense

$$
-W_{t} \operatorname{det}\left(D^{2} W\right) \geq 1 \quad \text { in } \mathbb{R}_{T}^{n+1}
$$

Fix some $R_{1}>0$ such that

$$
\Omega \subset \subset B_{R_{1}}
$$

Let $R_{2}=2 R_{1}$. For $a>1$, define

$$
v_{a}(x, t):=-t+\inf _{B_{R_{1}} \times(0, T]} W+\int_{2 R_{2}}^{|x|}\left(s^{n}+a\right)^{\frac{1}{n}} d s, \quad(x, t) \in \mathbb{R}_{T}^{n+1}
$$

Then

$$
D_{i j} v_{a}=\left(|x|^{n}+a\right)^{\frac{1}{n}-1}\left[\left(|x|^{n-1}+\frac{a}{|x|}\right) \delta_{i j}-\frac{a x_{i} x_{j}}{|x|^{3}}\right], \quad|x|>0 .
$$

In virtue of the fact that for any constants $a$ and $b$, the eigenvalues of the symmetric matrix of the form $a x^{T} x+b I$ are

$$
\lambda\left(a x^{T} x+b I\right)=\left(a|x|^{2}+b, b, \ldots, b\right),
$$


then we have

$$
D^{2} v_{a}=\left(R^{n}+a\right)^{\frac{1}{n}-1}\left(\begin{array}{cccc}
R^{n-1} & 0 & \cdots & 0 \\
0 & R^{n-1}+\frac{a}{R} & \cdots & 0 \\
\cdots & \cdots & \cdots & \cdots \\
0 & 0 & \cdots & R^{n-1}+\frac{a}{R}
\end{array}\right)
$$

where $R=|x|$. So $v_{a}$ is parabolically convex and satisfies

$$
-\left(v_{a}\right)_{t} \operatorname{det}\left(D^{2} v_{a}\right)=1, \quad|x|>0,0 \leq t \leq T .
$$

Moreover, from the definition of $R_{2}$,

$$
\begin{aligned}
v_{a}(x, t) & \leq \inf _{B_{R_{1}} \times(0, T]} W+\int_{2 R_{2}}^{\frac{R_{2}}{2}}\left(s^{n}+a\right)^{\frac{1}{n}} d s \\
& <\inf _{B_{R_{1}} \times(0, T]} W \\
& \leq W(x, t), \quad|x| \leq R_{1}, 0 \leq t \leq T .
\end{aligned}
$$

Fix some $R_{3}>3 R_{2}$ and choose $a_{1}>1$ such that for $a \geq a_{1}$,

$$
\begin{aligned}
v_{a}(x, t) & >-t+\inf _{B_{R_{1}} \times(0, T]} W+\int_{2 R_{2}}^{3 R_{2}}\left(s^{n}+a\right)^{\frac{1}{n}} d s \\
& \geq W(x, t), \quad|x|=R_{3}, 0 \leq t \leq T .
\end{aligned}
$$

It is easy to see that

$$
\begin{aligned}
v_{a}(x, t)= & -t+\inf _{B_{R_{1}} \times(0, T]} W+\int_{2 R_{2}}^{|x|} s\left(\left(1+\frac{a}{s^{n}}\right)^{\frac{1}{n}}-1\right) d s+\int_{2 R_{2}}^{|x|} s d s \\
= & -t+\inf _{B_{R_{1}} \times(0, T]} W+\int_{2 R_{2}}^{|x|} s\left(\left(1+\frac{a}{s^{n}}\right)^{\frac{1}{n}}-1\right) d s+\frac{1}{2}|x|^{2}-2 R_{2}^{2} \\
= & -t+\frac{1}{2}|x|^{2}+c_{m}+\inf _{B_{R_{1}} \times(0, T]} W+\int_{2 R_{2}}^{\infty} s\left(\left(1+\frac{a}{s^{n}}\right)^{\frac{1}{n}}-1\right) d s-c_{m} \\
& -2 R_{2}^{2}-\int_{|x|}^{\infty} s\left(\left(1+\frac{a}{s^{n}}\right)^{\frac{1}{n}}-1\right) d s, \quad(x, t) \in \mathbb{R}_{T}^{n+1} .
\end{aligned}
$$

Let

$$
\mu(m, a)=\inf _{B_{R_{1}} \times(0, T]} W+\int_{2 R_{2}}^{\infty} s\left(\left(1+\frac{a}{s^{n}}\right)^{\frac{1}{n}}-1\right) d s-c_{m}-2 R_{2}^{2} .
$$

As a result,

$$
v_{a}(x, t)=-t+\frac{1}{2}|x|^{2}+c_{m}+\mu(m, a)-O\left(|x|^{2-n}\right), \quad \text { when }|x| \rightarrow \infty .
$$


For $a \geq a_{1}$ and $1 \leq m \leq k$, define

$$
\underline{u}_{m, a}(x, t)= \begin{cases}\max \left\{W(x, t), v_{a}(x, t)\right\}-\mu(m, a), & |x| \leq R_{3}, 0 \leq t \leq T \\ v_{a}(x, t)-\mu(m, a), & |x| \geq R_{3}, 0 \leq t \leq T\end{cases}
$$

From (3.6), we have

$$
\underline{u}_{m, a}(x, t)=-t+\frac{1}{2}|x|^{2}+c_{m}-O\left(|x|^{2-n}\right), \quad \text { when }|x| \rightarrow \infty .
$$

By the definition of $W$ and (3.2), (3.4), (3.5),

$$
\underline{u}_{m, a}(x, t)=-t_{0}+\frac{1}{2}-\mu(m, a), \quad(x, t) \in \Gamma .
$$

Because $\mu(m, a)$ is continuous and monotonic increasing in $a$ and for $a \rightarrow \infty, \mu(m, a) \rightarrow$ $\infty, 1 \leq m \leq k$, we can choose $a_{2} \geq a_{1}$ sufficiently large such that for $a \geq a_{2}$,

$$
\begin{aligned}
W(x, t)-\mu(m, a) & =W(x, t)-\inf _{B_{R_{1}} \times(0, T]} W-\int_{2 R_{2}}^{\infty} s\left(\left(1+\frac{a}{s^{n}}\right)^{\frac{1}{n}}-1\right) d s+c_{m}+2 R_{2}^{2} \\
& \leq-T+c_{m} \leq-t+\frac{1}{2}|x|^{2}+c_{m}, \quad|x| \leq R_{3}, 0 \leq t \leq T .
\end{aligned}
$$

Clearly,

$$
v_{a}(x, t)-\mu(m, a) \leq-t+\frac{1}{2}|x|^{2}+c_{m}, \quad a \geq a_{2},(x, t) \in \mathbb{R}_{T}^{n+1} .
$$

Therefore

$$
\underline{u}_{m, a}(x, t) \leq-t+\frac{1}{2}|x|^{2}+c_{m}, \quad a \geq a_{2},(x, t) \in \mathbb{R}_{T}^{n+1}
$$

By Lemma $2.1, \underline{u}_{m, a} \in C^{0}\left(\mathbb{R}_{T}^{n+1}\right)$ is parabolically convex and satisfies in the viscosity sense

$$
-\left(\underline{u}_{m, a}\right)_{t} \operatorname{det}\left(D^{2} \underline{u}_{m, a}\right) \geq 1 \quad \text { in } \mathbb{R}_{T}^{n+1} \text {. }
$$

It is clear that there exists a continuous function $a^{(m)}(a), 2 \leq m \leq k$, satisfying

$$
\lim _{a \rightarrow \infty} a^{(m)}(a)=\infty
$$

and, for $2 \leq m \leq k$,

$$
\mu\left(m, a^{(m)}(a)\right)=\mu(1, a)
$$

Thus there exists $a_{3} \geq a_{2}$ such that for $a \geq a_{3}, a^{(m)}(a)>a_{2}, 2 \leq m \leq k$. Set $a^{(1)}(a)=a$, and define

$$
\underline{u}_{a}(x, t, m)=\underline{u}_{m, a^{(m)}(a)}(x, t), \quad(x, t, m) \in G_{k} .
$$


Then for $a \geq a_{3}, \underline{u}_{a} \in C^{0}\left(G_{k}\right)$ is locally parabolically convex and satisfies

$$
\begin{aligned}
& \underline{u}_{a}(x, t, m)=-t+\frac{1}{2}|x|^{2}+c_{m}-O\left(|x|^{2-n}\right), \quad|x| \rightarrow \infty, 0 \leq t \leq T, \\
& \underline{u}_{a}(x, t, m) \leq-t+\frac{1}{2}|x|^{2}+c_{m}, \quad(x, t) \in \mathbb{R}_{T}^{n+1} \backslash \Gamma, \\
& \lim _{(x, t) \rightarrow\left(\bar{x}, t_{0}\right)} \underline{u}_{a}(x, t, m)=-t_{0}+\frac{1}{2}-\mu(1, a), \quad\left(\bar{x}, t_{0}\right) \in \Gamma,
\end{aligned}
$$

and in the viscosity sense,

$$
-\left(\underline{u}_{a}\right)_{t} \operatorname{det}\left(D^{2} \underline{u}_{a}\right) \geq 1, \quad(x, t, m) \in G_{k} .
$$

Step 2. We define the Perron solution of (1.2).

For $a \geq a_{3}$, let $\mathbb{S}_{a}$ denote the set of locally parabolically convex functions $v \in C^{0}\left(G_{k}\right)$ which can be extended to $\Gamma$ and satisfy

$$
\begin{aligned}
& -v_{t} \operatorname{det}\left(D^{2} v\right) \geq 1, \quad(x, t, m) \in G_{k}, \\
& \limsup _{(x, t) \rightarrow\left(\bar{x}, t_{0}\right)} v(x, t, m) \leq-t_{0}+\frac{1}{2}-\mu(1, a), \quad\left(\bar{x}, t_{0}\right) \in \Gamma, \\
& v(x, t, m) \leq-t+\frac{1}{2}|x|^{2}+c_{m}, \quad(x, t) \in \mathbb{R}_{T}^{n+1} \backslash \Gamma, 1 \leq m \leq k .
\end{aligned}
$$

Apparently, $\underline{u}_{a} \in \mathbb{S}_{a}$ and so $\mathbb{S}_{a} \neq \emptyset$. Define

$$
u_{a}(x, t, m):=\sup \left\{v(x, t, m) \mid v \in \mathbb{S}_{a}\right\}, \quad(x, t, m) \in G_{k} .
$$

Step 3. We prove that $u_{a}$ is a viscosity solution of (1.2).

By the definition of $u_{a}$ and Lemma 2.3, $u_{a}$ is a viscosity subsolution of (1.2). We only need to prove that $u_{a}$ is a viscosity supersolution of (1.2). For any $(\tilde{x}, \tilde{t}) \in \mathbb{R}_{T}^{n+1} \backslash \Gamma$, fix some $r>0$ such that $0<\tilde{t}-r<\tilde{t}<\tilde{t}+r \leq T$ and $Q_{r}=B_{r}(\tilde{x}) \times(\tilde{t}-r, \tilde{t}+r] \subset Q \backslash \Gamma$. Then the lifting of $Q_{r}$ into $G_{k}$ is the union of $k$ disjoint cylinders denoted as $\left\{Q_{r}^{(i)}\right\}_{i=1}^{k}$. In each $Q_{r}^{(i)}$, by Theorem B in [9], the Dirichlet problem

$$
\begin{aligned}
& -\varphi_{t} \operatorname{det}\left(D^{2} \varphi\right)=1 \quad \text { in } Q_{r}^{(i)}, \\
& \varphi=u_{a} \quad \text { on } \partial_{p} Q_{r}^{(i)}
\end{aligned}
$$

has a parabolically convex viscosity solution $\varphi \in C^{0}\left(\bar{Q}_{r}^{(i)}\right)$. According to the comparison principle, we have

$$
u_{a} \leq \varphi \quad \text { in } Q_{r}^{(i)}
$$

Define

$$
h(x, t, m)= \begin{cases}\varphi(x, t, m) & \text { in } Q_{r}^{(i)}, \\ u_{a}(x, t, m) & \text { in } G_{k} \backslash\left\{Q_{r}^{(i)}\right\}_{i=1}^{k} .\end{cases}
$$


Next we prove that $h \in \mathbb{S}_{a}$. Then $u_{a} \geq h$ in $G_{k}$ and so $u_{a} \geq \varphi$ in $Q_{r}^{(i)}$. By (3.9), $u_{a}=\varphi$ in $Q_{r}^{(i)}$. Consequently by the arbitrariness of $(\tilde{x}, \tilde{t})$, we know that $u_{a}$ is a viscosity solution of (1.2).

From Lemma $2.1, h$ is a viscosity subsolution of (1.2). In order to prove $h \in \mathbb{S}_{a}$, we only need to prove

$$
\varphi \leq-t+\frac{1}{2}|x|^{2}+c_{m} \quad \text { in } Q_{r}^{(i)} .
$$

In fact, we have

$$
\begin{aligned}
& -\varphi_{t} \operatorname{det}\left(D^{2} \varphi\right)=1=-g_{t} \operatorname{det}\left(D^{2} g\right) \text { in } Q_{r}^{(i)}, \\
& \varphi=u_{a} \leq g \quad \text { on } \partial_{p} Q_{r}^{(i)}
\end{aligned}
$$

where

$$
g(x, t, m)=-t+\frac{1}{2}|x|^{2}+c_{m} .
$$

From the comparison principle,

$$
\varphi \leq g \quad \text { on } \bar{Q}_{r}^{(i)} .
$$

That is,

$$
\varphi \leq-t+\frac{1}{2}|x|^{2}+c_{m}
$$

Thus (3.10) holds.

Step 4. We prove that (1.3) holds.

By the definition of $u_{a}$,

$$
\underline{u}_{a} \leq u_{a} \leq-t+\frac{1}{2}|x|^{2}+c_{m} .
$$

From (3.7), we have for $|x| \rightarrow \infty$,

$$
-t+\frac{1}{2}|x|^{2}+c_{m}-O\left(|x|^{2-n}\right) \leq u_{a} \leq-t+\frac{1}{2}|x|^{2}+c_{m}+O\left(|x|^{2-n}\right) .
$$

Therefore (1.3) holds.

Step 5. We prove that $u_{a}$ satisfies (1.4).

Because $\underline{u}_{a} \in \mathbb{S}_{a}$ and

$$
\lim _{(x, t) \rightarrow\left(\bar{x}, t_{0}\right)} \underline{u}_{a}=-t_{0}+\frac{1}{2}-\mu(1, a),
$$

then

$$
\liminf _{(x, t) \rightarrow\left(\bar{x}, t_{0}\right)} u_{a}(x, t, m) \geq-t_{0}+\frac{1}{2}-\mu(1, a), \quad\left(\bar{x}, t_{0}\right) \in \Gamma
$$


In the following, we prove

$$
\limsup _{(x, t) \rightarrow\left(\bar{x}, t_{0}\right)} u_{a}(x, t, m) \leq-t_{0}+\frac{1}{2}-\mu(1, a), \quad\left(\bar{x}, t_{0}\right) \in \Gamma .
$$

Let $v \in \mathbb{S}_{a},\left(\bar{x}, t_{0}\right) \in \Gamma$, and $(x, t) \in \mathcal{N}\left(\bar{x}, t_{0}\right), \mathcal{N}\left(\bar{x}, t_{0}\right)$ be the neighborhood of $\left(\bar{x}, t_{0}\right)$. Suppose $\left(x_{i}, t\right) \in \mathcal{N}\left(\bar{x}, t_{0}\right)$ and $x_{i} \rightarrow \bar{x}$, then by the convexity of $v$ in $x$, we have, for some constant $C$,

$$
v(x, t, m)-v\left(x_{i}, t, m\right) \leq C\left\|x-x_{i}\right\| .
$$

Then

$$
v(x, t, m) \leq v\left(x_{i}, t, m\right)+C\left\|x-x_{i}\right\| .
$$

Thus

$$
u_{a}(x, t, m) \leq v\left(x_{i}, t, m\right)+C\left\|x-x_{i}\right\| .
$$

Sending $i$ to infinity, we have

$$
u_{a}(x, t, m) \leq-t_{0}+\frac{1}{2}-\mu(1, a)+C\|x-\bar{x}\|
$$

It follows that (3.12) holds. Choose $\beta=-t_{0}+\frac{1}{2}-\mu(1, a)$, then (1.4) is true with this $\beta$. Theorem 1.1 is proved.

\section{Competing interests}

The author declares that there are no competing interests.

\section{Acknowledgements}

The author is indebted to the referees for their helpful comments. This work is supported by National Natural Science Foundation of China (11201343), Shandong Province Young and Middle-Aged Scientists Research Awards Fund (BS2011SF025)

Received: 20 September 2014 Accepted: 17 February 2015 Published online: 04 March 2015

\section{References}

1. Krylov, NV: Sequences of convex functions, and estimates of the maximum of the solution of a parabolic equation. Sib. Mat. Zh. 17, 290-303 (1976) (Russian)

2. Lieberman, GM: Second Order Parabolic Differential Equations. World Scientific, River Edge (1996)

3. Krylov, NV: Boundedly inhomogeneous elliptic and parabolic equations. Izv. Akad. Nauk SSSR, Ser. Mat. 46, 487-523 (1982) (Russian)

4. Tso, K: Deforming a hypersurface by its Gauss-Kronecker curvature. Commun. Pure Appl. Math. 38, 867-882 (1985)

5. Tso, K: On an Aleksandrov-Bakelman type maximum principle for second-order parabolic equations. Commun. Partial Differ. Equ. 10, 543-553 (1985)

6. Gutiérrez, CE, Huang, QB: W2,p Estimates for the parabolic Monge-Ampère equation. Arch. Ration. Mech. Anal. 159, 137-177 (2001)

7. Gutiérrez, CE, Huang, QB: A generalization of a theorem by Calabi to the parabolic Monge-Ampère equation. Indiana Univ. Math. J. 47, 1459-1480 (1998)

8. Lian, S: Existence of solutions to initial value problem for a parabolic Monge-Ampère equation and application. Nonlinear Anal. 65, 59-78 (2006)

9. Wang, $\mathrm{RH}$, Wang, GL: On the existence, uniqueness and regularity of viscosity solution for the first initial boundary value problem to parabolic Monge-Ampère equations. Northeast. Math. J. 8, 417-446 (1992)

10. Wang, $\mathrm{RH}$, Wang, GL: The geometric measure theoretical characterization of viscosity solutions to parabolic Monge-Ampère type equation. J. Partial Differ. Equ. 6, 237-254 (1993) 
11. Xiong, JG, Bao, JG: On Jörgens, Calabi, and Pogorelov type theorem and isolated singularities of parabolic Monge-Ampère equations. J. Differ. Equ. 250, 367-385 (2011)

12. Evans, GC: Surfaces of minimal capacity. Proc. Natl. Acad. Sci. USA 26, 489-491 (1940)

13. Caffarelli, L: On the Hölder continuity of multiple valued harmonic functions. Indiana Univ. Math. J. 25, 79-84 (1976)

14. Jin, S, Liu, H, Osher, S, Tsai, Y: Computing multivalued physical observables for the semiclassical limit of the Schrödinger equation. J. Comput. Phys. 205, 222-241 (2005)

15. Jin, S, Osher, S: A level set method for the computation of multi-valued solutions to quasi-linear hyperbolic PDE's and Hamilton-Jacobi equations. Commun. Math. Sci. 1, 575-591 (2003)

16. Jin, S, Li, X: Multi-phase computations of the semiclassical limit of the Schrödinger equation and related problems: Whitham vs. Wigner. Physica D 182, 46-85 (2003)

17. Caffarelli, L, Li, YY: Some multi-valued solutions to Monge-Ampère equations. Commun. Anal. Geom. 14, 411-441 (2006)

18. Dai, LM, Bao, JG: Multi-valued solutions to Hessian equations. Nonlinear Differ. Equ. Appl. 18, 447-457 (2011)

19. Dai, LM: Multi-valued solutions to a class of parabolic Monge-Ampère equations. Commun. Pure Appl. Anal. 13, 1061-1074 (2014)

20. Ghergu, M, Radulescu, V: Nonlinear PDEs: Mathematical Models in Biology, Chemistry and Population Genetics. Springer Monographs in Mathematics. Springer, Heidelberg (2012)

\section{Submit your manuscript to a SpringerOpen ${ }^{\circ}$ journal and benefit from:}

- Convenient online submission

- Rigorous peer review

- Immediate publication on acceptance

- Open access: articles freely available online

High visibility within the field

- Retaining the copyright to your article 